\title{
SOME PROPERTIES OF CONTINUED FRACTIONS
}

$$
1+d_{0} z+K\left(z /\left(1+d_{n} z\right)\right)
$$

\section{W. J. THRON}

1. Introduction. A continued fraction

$$
b_{0}(z)+\frac{a_{1}(z)}{b_{1}(z)}+\frac{a_{2}(z)}{b_{2}(z)}+\cdots,
$$

with $n$th approximant $A_{n}(z) / B_{n}(z)$, is said to correspond to the power series

$$
1+c_{1} z+c_{2} z^{2}+\cdots
$$

if the power series expansion of $A_{n}(z) / B_{n}(z)$ agrees with (1.2) up to and including the term $c_{k(n)} z^{k(n)}$, where $k(n) \rightarrow \infty$ as $n \rightarrow \infty$.

Leighton and Scott $[1]^{1}$ proved that there is one and only one continued fraction of the form

$$
1+\frac{a_{1} z^{k_{1}}}{1}+\frac{a_{2} z^{k_{2}}}{1}+\cdots,
$$

where all $k_{n}$ are positive integers, which corresponds to a given power series (1.2).

The class of all continued fractions of the form

$$
1+d_{0} z+\frac{z}{1+d_{1} z}+\frac{z}{1+d_{2} z}+\cdots,
$$

which is studied in this paper, has the same property. This is shown in $\$ 2$. In $\$ 3$ convergence, and in particular uniform convergence, of continued fractions (1.4) is investigated. $\$ 4$, finally, is devoted to a study of necessary conditions for the uniform convergence of (1.4) in a neighborhood of the origin.

2. Correspondence. A sufficient condition for the existence of a continued fraction (1.4) which corresponds to a given power series $P(z)$ of the form (1.2) is the existence of a solution for the system of formal identities

$$
P_{n}(z) \equiv 1+d_{n} z+\frac{z}{P_{n+1}(z)}, \quad n=0,1, \cdots,
$$

Presented to the Society, September 4, 1947; received by the editors March 19, 1947, and, in revised form, April 23, 1947.

1 Numbers in brackets refer to the bibliography at the end of the paper. 
where $P_{0}(z) \equiv P(z)$ and $P_{n}(z)$ is a formal power series of the form (1.2). This is seen as follows. If such a solution exists one has for every $n \geqq 1$

$$
P(z) \equiv 1+d_{0} z+\frac{z}{1+d_{1} z}+\cdots+\frac{z}{1+d_{n-1} z}+\frac{z}{P_{n}(z)} .
$$

Hence

$$
P(z) \equiv \frac{P_{n}(z) \cdot A_{n-1}(z)+z \cdot A_{n-2}(z)}{P_{n}(z) \cdot B_{n-1}(z)+z \cdot B_{n-2}(z)}
$$

and therefore

$$
P(z)-\frac{A_{n-1}(z)}{B_{n-1}(z)} \equiv \frac{(-1)^{n-1} z^{n}}{B_{n-1}(z)\left[P_{n}(z) \cdot B_{n-1}(z)+z \cdot B_{n-2}(z)\right]} .
$$

Since $B_{n}(0)$ and $P_{n}(0)$ are both different from zero for all $n$ one now concludes that the power series expansion of the $n$th approximant agrees with $P(z)$ up to and including the term $c_{n} z^{n}$. The continued fraction therefore corresponds to $P(z)$.

Let $P_{n}^{\prime}(z)$ be the formal derivative of $P_{n}(z)$. It is easily verified that $d_{n}=P_{n}^{\prime}(0)-1$ and

$$
P_{n+1}(z) \equiv \frac{z}{P_{n}(z)-1-d_{n} z}
$$

provides a solution of the system (2.1). The following theorem has now been proved.

THEOREM 2.1. For every power series of the form

$$
1+c_{1} z+c_{2} z^{2}+\cdots
$$

there exists a continued fraction of the form

$$
1+d_{0} z+\frac{z}{1+d_{1} z}+\frac{z}{1+d_{2} z}+\cdots
$$

which corresponds to the power series. The power series expansion of the nth approximant of the continued fraction agrees with the given power series up to and including the term $c_{n} z^{n}$.

The question whether more than one continued fraction of the type (1.4) can correspond to the same power series is answered in the next theorem.

THEOREM 2.2. Two continued fractions 


$$
\begin{aligned}
& 1+d_{0} z+\stackrel{\infty}{K}_{\nu=1}^{\infty}\left(\frac{z}{1+d_{\nu} z}\right) \\
& 1+d_{0}^{\prime} z+\stackrel{\infty}{K}_{\nu=1}^{\infty}\left(\frac{z}{1+d_{\nu}^{\prime} z}\right)
\end{aligned}
$$

correspond to the same power es

$$
1+c+c_{2} z^{2}+\cdots
$$

if and only if $d_{n}=d_{n}^{\prime}$ for all $n \geqq 0$.

Let $A_{n} / B_{n}$ and $A_{n}^{\prime} / B_{n}^{\prime}$ be the $n$th approximants of the continued fractions (1) and (2) respectively. If both continued fractions correspond to the same power series then their $(n+1)$ th approximants have power series expansions which certainly agree with each other up to and including the term involving $z^{n+1}$. Now

$$
\frac{A_{n}}{B_{n}}-\frac{A_{n+1}}{B_{n+1}} \equiv(-1)^{n+1} z^{n+1}+\cdots,
$$

and similarly

$$
\frac{A_{n}^{\prime}}{B_{n}^{\prime}}-\frac{A_{n+1}^{\prime}}{B_{n+1}^{\prime}} \equiv(-1)^{n+1} z^{n+1}+\cdots .
$$

It follows that the power series expansions of $A_{n}^{\prime} / B_{n}^{\prime}$ and $A_{n} / B_{n}$ agree up to and including the term involving $z^{n+1}$. Now $B_{0}=B_{0}^{\prime}=1$ and hence $A_{0} \equiv A_{0}^{\prime}$ and $d_{0}=d_{0}^{\prime}$.

The proof of the theorem is then complete if it is shown that the assumption

$$
A_{\nu} \equiv A_{\nu}^{\prime}, \quad B_{\nu} \equiv B_{\nu}^{\prime} \text { and hence } d_{\nu}=d_{\nu}^{\prime}
$$

for $\nu=0,1, \cdots, m-1$, leads to the conclusion $d_{m}=d_{m}{ }^{\prime}$ and $A_{m} \equiv A_{m}{ }^{\prime}$, $B_{m} \equiv B_{m}^{\prime}$. Making those assumptions one obtains

$$
\begin{aligned}
\frac{A_{m}}{B_{m}}-\frac{A_{m}^{\prime}}{B_{m}^{\prime}} & \equiv \frac{A_{m} B_{m}^{\prime}-A_{m}^{\prime} B_{m}}{B_{m} B_{m}^{\prime}} \\
& \equiv \frac{z^{2}\left(d_{m}-d_{m}^{\prime}\right)\left(A_{m-1} B_{m-2}-A_{m-2} B_{m-1}\right)}{B_{m} B_{m}^{\prime}} \\
& \equiv \frac{(-1)^{m-2} z^{m+1}\left(d_{m}-d_{m}^{\prime}\right)}{B_{m} B_{m}^{\prime}} .
\end{aligned}
$$

A combination of this result with the one concerning the power series 
expansion of this difference leads to the conclusion $d_{m}=d_{m}^{\prime}$ and hence $A_{m} \equiv A_{m}^{\prime}, B_{m} \equiv B_{m}^{\prime}$.

The proof of the theorem below is omitted since it is analogous to the proof given for the corresponding theorem concerning continued fractions $1+K\left(a_{n} z / 1\right)$ by Perron [2, p. 342].

THEOREM 2.3. If the continued fraction (1.4) converges uniformly in a region $D$, containing the origin in its interior, then the corresponding power series converges to the same function in the largest open circular region, with center at the origin, completely contained in $D$.

It is of course not implied that the power series converges only in that circle. It is not possible to deduce convergence of the continued fraction from the convergence of the power series. This is true for all types of "corresponding" continued fractions studied so far. (See [2, $\$ 65]$.) For the type studied here this phenomenon occurs for a very simple function. Contrary to the cases previously investigated there are no terminating expansions for this type of continued fraction. Even rational functions have nonterminating expansions. The expansion for $P(z) \equiv 1$ is easily seen to be

$$
1-z+\frac{z}{1-z}+\frac{z}{1-z}+\cdots .
$$

This continued fraction converges to 1 only for $|z|<1$, while it diverges for $|z|=1, z \neq-1$ and converges to $-z$ for all other values of $z$.

The final two theorems of this section are analogues of two theorems of Leighton and Scott [1, pp. 600-601] for continued fractions (1.3). The proofs in our case are almost identical to the proofs given there and are therefore omitted.

THEOREM 2.4. A necessary and sufficient condition that the continued fraction (1.4) converge uniformly to a holomorphic function in an open region $D$, containing the origin, is that the approximants $A_{n}(z) / B_{n}(z)$ be uniformly bounded in every closed set contained in $D$ for all $n>n_{0}$.

THEOREM 2.5. Two infinite subsequences of approximants of the continued fraction (1.4) which converge uniformly in an open region $D$, containing the origin, converge to the same holomorphic function in $D$.

3. Convergence criteria. It is well known [2, p. 196] that for $z \neq 0$ the continued fractions (1.4) and

$$
x+d_{0} / x+\frac{1}{x+d_{1} / x}+\frac{1}{x+d_{2} / x}+\cdots,
$$


where $x=z^{-1 / 2},-\pi / 2<\arg x \leqq \pi / 2$, converge and diverge together. The expression (3.1) is of the form $b_{0}+K\left(1 / b_{n}\right)$, where

$$
b_{n}=x+d_{n} / x
$$

and hence

$$
d_{n}=x b_{n}-x^{2}
$$

Convergence criteria developed for continued fractions of the form $b_{0}+K\left(1 / b_{n}\right)$ (see [3]) are therefore applicable to (3.1) and consequently indirectly to (1.4).

Conditions on the sequence $\left\{d_{n}\right\}$ which insure convergence of (1.4) in a neighborhood of the point $z=0$ can be obtained as follows. Let $\left|d_{n}\right| \leqq M$ for all $n$ and let $|x|=r$. Then

$$
\left|b_{n}\right| \geqq r-M / r \text {. }
$$

Now for $r \geqq 1+(M+1)^{1 / 2}$ one has $r-M / r \geqq 2$ and hence $\left|b_{n}\right| \geqq 2$. The Pringsheim criterion then insures the convergence of (3.1). It follows from the equivalence that the continued fraction (1.4) converges for all $z \neq 0$ for which

$$
|z| \leqq 1 /\left(1+(M+1)^{1 / 2}\right)^{2} .
$$

The convergence of (1.4) for $z=0$ is trivial. For all values of $z$ under consideration the approximants of (1.4) are uniformly bounded. This is seen as follows:

$$
\left|\prod_{\nu=1}^{n}\left(\frac{z}{1+d_{\nu} z}\right)\right|=\left|\frac{1}{x}\right| \cdot\left|\prod_{\nu=1}^{n}\left(\frac{1}{x+d_{\nu} / x}\right)\right| \leqq \frac{1}{1+(M+1)^{1 / 2}} \cdot 1,
$$

since, for $\left|x+d_{n} / x\right| \geqq 2,\left|K\left(1 /\left(x+d_{\nu} / x\right)\right)\right| \leqq 1$ [3, Theorem 3.2]. An application of the Vitali theorem then leads to the conclusion that (1.4) converges uniformly in every closed set contained in the circular region

$$
z<1 /\left(1+(M+1)^{1 / 2}\right)^{2} .
$$

THEOREM 3.1. If

$$
\left|d_{n}\right| \leqq M
$$

for all $n$ then the continued fraction (1.4) converges for all $z$ in the circular region

$$
|z| \leqq 1 /\left(1+(M+1)^{1 / 2}\right)^{2}
$$

The convergence is uniform in every closed set contained in the interior of this circular region. 
Observing that if $\left|d_{n}\right| \geqq m$ then

$$
\left|b_{n}\right| \geqq m / r-r
$$

one easily shows that (1.4) converges if

$$
|z| \geqq 1 /\left(1-(m+1)^{1 / 2}\right)^{2} \text {. }
$$

To show that the convergence is uniform one makes use of the fact that in this case the approximants of (1.4) are uniformly bounded away from the origin but are finite. One thus proves the following result.

Theorem 3.2. If

$$
\left|d_{n}\right| \geqq m
$$

for all $n$ then the continued fraction (1.4) converges for all $z$ in the region

$$
|z| \geqq 1 /\left(1-(m+1)^{1 / 2}\right)^{2} .
$$

The convergence is uniform in every closed bounded set in the interior of this region.

These theorems gain in importance if they are used in conjunction with the following results which give criteria for the convergence of (1.4) in certain angular openings.

THEOREM 3.3. Let the region $D(\gamma, k, \epsilon)$ be defined by: $r \cdot e^{i \theta}$ $\in D(\gamma, k, \epsilon)$ if

$$
r \geqq \frac{2+\epsilon}{\left(c^{2}-k^{2}\right)^{1 / 2}-c \cdot \cos \left(\gamma^{\prime}-\theta\right)},
$$

where $-\pi / 2<\gamma \leqq \pi / 2, k<1 / 2 \cos \gamma, \epsilon>0$, and

$$
\begin{aligned}
& \gamma=\arg \left(k \cdot e^{i \gamma}-e^{2 i \gamma}\right), \\
& c=\left|k e^{i \gamma}-e^{2 i \gamma}\right| .
\end{aligned}
$$

If there exist functions $\epsilon(\gamma)$ and $k(\gamma)$ and constants $\gamma_{1}$ and $\gamma_{2}$ such that all elements of the sequence $\left\{d_{n}\right\}$ lie in the intersections of the regions $D(\gamma, k(\gamma), \epsilon(\gamma))$ for $\gamma_{1} \leqq \gamma \leqq \gamma_{2}$ then the continued fraction (1.4) converges for all $z$ in the angular opening

$$
-2 \gamma_{2} \leqq \arg z \leqq-2 \gamma_{1} .
$$

Moreover the convergence is uniform in every closed bounded set in the interior of this region.

Consider a fixed $x=\rho e^{i \gamma}$ and define as $C(\rho)$ the region formed by 
all points on or outside the circle with center at

$$
\rho^{2}\left(k e^{i \gamma}-e^{2 i \gamma}\right)
$$

and radius

$$
\rho\left(4+2 \epsilon+k^{2} \rho^{2}\right)^{1 / 2},
$$

where $\epsilon$ is positive and $k$ is real.

Assume that all numbers $d_{n}$ of (3.1) lie in the region $C(\rho)$. Then the elements $b_{n}$ of (3.1), connected with the numbers $d_{n}$ by relation (3.2), satisfy the condition

$$
\left|b_{n}-k \rho\right| \geqq\left(4+2 \epsilon+k^{2} \rho^{2}\right)^{1 / 2} .
$$

For every fixed $\rho$ therefore

$$
\left|b_{n}-k \rho\right| \geqq\left(4+k^{2} \rho^{2}\right)^{1 / 2}+\eta(\rho),
$$

where $\eta(\rho)>0$. A known result [3, Corollary 6.2] then insures the convergence of (3.1) for for $x=\rho e^{i \gamma}$.

If all the elements of the sequence $\left\{d_{n}\right\}$ lie in a region $D(\gamma, k, \epsilon)$ which is the intersection of all sets $C(\rho), 0<\rho<\infty$, then it follows from what was said above that the continued fraction (3.1) converges for every $x \neq 0$ on the ray arg $x=\gamma$. The boundary of $D(\gamma, k, \epsilon)$ is the envelope of the boundaries of the regions $C(\rho)$.

Recalling the definition of $\gamma^{\prime}$, noting that

$$
c=\left|k e^{i \gamma}-e^{2 i \gamma}\right|=\left(k^{2}+1-2 k \cdot \cos \gamma\right)^{1 / 2},
$$

and denoting the boundary points of $C(\rho)$ by $r \cdot e^{i \theta}$ one easily arrives at the following equation for the boundary of $C(\rho)$

$$
\begin{gathered}
r^{2}+\rho^{4}\left(k^{2}+1-2 k \cdot \cos \gamma\right)-2 r \rho^{2} \cos \left(\gamma^{\prime}-\theta\right)\left(k^{2}+1-2 k \cos \gamma\right)^{1 / 2} \\
=(4+2 \epsilon) \rho^{2}+k^{2} \rho^{4} .
\end{gathered}
$$

Combination of like powers of $\rho$ and introduction of $c$ leads to

$$
r^{2}+\rho^{4}\left(c^{2}-k^{2}\right)-2 \rho^{2}\left(r c \cdot \cos \left(\gamma^{\prime}-\theta\right)+2+\epsilon\right)=0 .
$$

Differentiating (3.5) with respect to $\rho^{2}$ one obtains

$$
\rho^{2}\left(c^{2}-k^{2}\right)=r c \cdot \cos \left(\gamma^{\prime}-\theta\right)+2+\epsilon .
$$

Elimination of $\rho$ from (3.5) leads to

$$
r^{2}\left(c^{2}-k^{2}\right)-\left(r c \cos \left(\gamma^{\prime}-\theta\right)+2+\epsilon\right)^{2}=0 .
$$

A part of the curve defined by this equation is the desired envelope. Thus one finds that $r \cdot e^{i \theta} \in D(\gamma, k, \epsilon)$ if and only if 


$$
r \geqq \frac{2+\epsilon}{\left(c^{2}-k^{2}\right)^{1 / 2}-c \cdot \cos \left(\gamma^{\prime}-\theta\right)} .
$$

The proof of the first part of Theorem 3.3 is now easily completed with the remark that the convergence of (3.1) implies the convergence of (1.4) for the corresponding $z \neq 0$. That (1.4) converges for $z=0$ is obvious.

From the results concerning value regions of [3] it follows that the imaginary numbers on the line segment from $-i$ to $i$ are not taken on by any of the approximants $\phi_{n}(x)$ of (3.1) for $\gamma_{1} \leqq \arg x \leqq \gamma_{2}, x \neq 0$. Further, the functions $\phi_{n}(x)$ are rational functions of $x$ which have no poles in the region under consideration [3, Lemma 2.2]. Thus the sequence $\left\{\phi_{n}(x)\right\}$ is a normal family of holomorphic functions in this region and since it converges it converges uniformly (Montel-Vitali theorem) in every closed bounded set in the interior of the region under consideration. Finally, if $\left\{f_{n}(z)\right\}$ is the sequence of approximants of (1.4) it follows from the relation

$$
f_{n}(z)=\frac{1}{x} \phi_{n}(x)
$$

that $\left\{f_{n}(z)\right\}$ converges uniformly wherever $\left\{\phi_{n}(x)\right\}$ does provided $|1 / x|$ or $|z|$ is bounded. This completes the proof of the theorem.

For $k=0$ the number $\epsilon$ can be omitted in the statement of Theorem 3.3 since (3.1) is known to converge if $\left|b_{n}\right| \geqq 2$. Further, if $k=0$ then $\gamma^{\prime}=\pi-2 \gamma$ and $c=1$. These remarks together with the substitution $\beta=-2 \gamma$ suffice to establish the following corollary of Theorem 3.3.

COROLlaRY 3.1. Let $P(\beta)$ be the region, not containing the origin, bounded by the parabola

$$
r=\frac{2}{1+\cos (\theta-\beta)}
$$

If there exists an interval $\left[\beta_{1}, \beta_{2}\right]$ such that all elements of the sequence $\left\{d_{n}\right\}$ lie in the intersection of the regions $P(\beta)$ as $\beta$ ranges over the interval $\left[\beta_{1}, \beta_{2}\right]$ then the continued fraction (1.4) converges for all $z$ in the angular opening

$$
\beta_{1} \leqq \arg z \leqq \beta_{2} .
$$

Moreover the convergence is uniform in every closed bounded set contained in the interior of this angular opening.

The result corresponding to the limiting case $k \rightarrow-\infty$ of Theorem 3.3 can be stated as follows. 
THEOREM 3.4. If there exist real numbers $\gamma$ and $\delta$, with $-\pi<\gamma<0$ $<\delta<\pi$ and $\delta-\gamma<\pi$, such that for suitable choice of the argument

$$
\gamma \leqq \arg d_{n} \leqq \delta \quad \text { for all } n
$$

then the continued fraction (1.4) converges for all $z$ in the angular opening

$$
-\pi-2 \gamma<\arg z<\pi-2 \delta .
$$

The convergence is uniform in every closed bounded set in the interior of this angular opening. ${ }^{2}$

Van Vleck's convergence criterion insures the convergence of (3.1) provided

$$
-\pi / 2+\epsilon \leqq \arg b_{n} \leqq \pi / 2-\epsilon,
$$

and provided further $\sum\left|b_{n}\right|$ diverges. Consider a fixed $x \neq 0$, such that

$$
-\pi / 2+\delta<\arg x<\pi / 2+\gamma .
$$

The corresponding $z$ then satisfies the conditions of the theorem. Now let

$$
\epsilon(x)=\min (\arg x+\pi / 2-\delta ; \pi / 2+\gamma-\arg x) ;
$$

then $\epsilon(x)>0$ and

$$
\begin{aligned}
-\pi / 2+\epsilon(x) & \leqq-\pi / 2+\delta+\epsilon(x) \\
& \leqq \arg x \leqq \pi / 2+\gamma-\epsilon(x) \leqq \pi / 2-\epsilon(x) .
\end{aligned}
$$

Further

$$
\begin{aligned}
-\pi / 2+\epsilon(x) & \leqq \gamma-\pi / 2-\gamma+\epsilon(x) \leqq \arg \left(d_{n} / x\right) \\
& \leqq \delta+\pi / 2-\delta-\epsilon(x) \leqq \pi / 2-\epsilon(x),
\end{aligned}
$$

provided the numbers $d_{n}$ satisfy the conditions of the theorem. It is then clear that the elements $b_{n}$, defined by relation (3.2), satisfy condition (3.6) with $\epsilon=\epsilon(x) . \sum\left|b_{n}\right|$ converges only if $\lim d_{n}=d$ and if $z=-1 / d$. This value however does not lie in the angular opening obtained for the variable $z$. This completes the proof of the convergence of (3.1) and hence of (1.4).

The uniform convergence is deduced in a manner analogous to that employed in the proof of Theorem 3.3. In this case the values of the approximants of (3.1) all lie in the half-plane $\Re(w) \geqq 0$.

${ }^{2}$ The original statement of this theorem contained an error which was kindly pointed out by the referee. 
All the preceding theorems of this section have the following common characteristics: For $\left\{d_{n}\right\}$ contained in a certain region $D$ there exists a region $Z(D)$ such that for all $z \in Z(D)$ the continued fraction (1.4) converges. Moreover we saw that the convergence is uniform over every closed bounded set in the interior of $Z(D)$. Since the approximants of (1.4) are rational functions and have no poles for $z \in Z(D)$ they are holomorphic for $z \in Z(D)$. The uniform convergence then insures that the limit function is holomorphic in the interior of $Z(D)$.

THEOREM 3.5. Let $D$ be any one of the regions defined in the preceding theorems such that for $\left\{d_{n}\right\} \subset D$ the continued fraction (1.4) converges uniformly for all $z$ contained in any arbitrary closed bounded set in the interior of the corresponding region $Z(D)$; then the function to which the continued fraction converges is holomorphic in the interior of $Z(D)$.

THEOREM 3.6. Let $L$ be the set of limit points of the sequence $\left\{d_{n}\right\}$, and let $S$ be an open set containing $L$. Let $D$ and $Z(D)$ be regions of the type defined in Theorem 3.5. If $S$ is contained in $D$ the continued fraction (1.4) converges, at least in the wider sense, for all $z \in Z(D)$ and the function to which it converges is meromorphic in the interior of $Z(D)$.

It is clear that there exists an integer $n^{\prime}$ such that, for all $n \geqq n^{\prime}$, $d_{n} \in S$. Thus Theorem 3.5 applies to the continued fraction

$$
1+d_{n^{\prime}} z+\frac{z}{1+d_{n^{\prime}+1} z}+\frac{z}{1+d_{n^{\prime}+2} z}+\cdots
$$

and Theorem 3.6 follows.

4. Behavior in a neighborhood of the origin. In Theorem 3.1 it was shown that the condition $\left|d_{n}\right| \leqq M$ insures uniform convergence of the continued fraction (1.4) in some neighborhood of the origin. This raises the question: to what extent is boundedness of the sequence $\left\{d_{n}\right\}$ a necessary condition for the existence of a neighborhood of the origin in which the continued fraction converges uniformly. Three partial answers to this question are given below.

TheOREM 4.1. If the sequence $\left\{d_{n} / n\right\}$ is unbounded then there does not exist a neighborhood of the origin in which the continued fraction (1.4) converges uniformly.

The denominator $B_{n}(z)$ of the $n$th approximant of (1.4) is a polynomial of $n$th degree

$$
B_{n}(z)=1+g_{1}^{(n)} z+\cdots+g_{n}^{(n)} z^{n} .
$$


From the recursion formula for $B_{n}(z)$, that is,

$$
B_{n}(z)=\left(1+d_{n} z\right) B_{n-1}(z)+z B_{n-2}(z),
$$

one easily deduces that

$$
g_{1}^{(n)}=n-1+\sum_{\nu=1}^{n} d_{\nu}
$$

Let $\rho_{\nu}^{(n)}$ be the roots of $B_{n}(z)$; then it is clear that

$$
g_{1}^{(n)}=-\sum_{\nu=1}^{n} \frac{1}{\rho_{\nu}(n)}
$$

One now shows that the sequence $\left\{g_{1}^{(n)} / n\right\}$ is unbounded. The following relation holds:

$$
\frac{g_{1}^{(n)}}{n}=\frac{d_{n}}{n}+\frac{1}{n}+\left(\frac{g_{1}^{(n-1)}}{n-1}\right)\left(\frac{n-1}{n}\right),
$$

and hence

$$
\left|\frac{g_{1}^{(n)}}{n}\right|+\left|\frac{g_{1}^{(n-1)}}{n-1}\right| \geqq\left|\frac{d_{n}}{n}\right| .
$$

The unboundedness of $g_{1}^{(n)} / n$ now follows immediately. Since $g_{1}^{(n)} / n$ is the arithmetic mean of the reciprocals of the roots one is now in a position to state that given an arbitrary positive $\delta$ and an arbitrary integer $N$ there exists an integer $N^{\prime}>N$ such that one of the roots of $B_{N^{\prime}}(z)$ lies in the neighborhood $|z|<\delta$. In order that there be uniform convergence in some neighborhood of the origin it is necessary that there exist a neighborhood of the origin in which $B_{n}(z) \neq 0$ for all $n>n_{0}$, since for $z=0$ (1.4) converges to 1 . This completes the proof of the theorem.

THEOREM 4.2. If $d_{n}>0$ for all $n$ and if $\left\{d_{n}\right\}$ is unbounded then there does not exist a neighborhood of the origin in which the continued fraction (1.4) converges uniformly.

The proof of this theorem depends on three lemmas which are also of some interest for their own sake.

Lemma 4.1. If $d_{\nu}>0$ for $\nu=0,1, \cdots, n$ then all the roots of $B_{n}(z)$ are real and negative and the roots of $B_{n}(z)$ and $B_{n-1}(z)$ separate each other, that is,

$$
\rho_{1}^{(n)}<\rho_{1}^{(n-1)}<\rho_{2}^{(n)}<\cdots<\rho_{n-1}^{(n-1)}<\rho_{n}^{(n)}<0 .
$$


One notes first that all the coefficients of $B_{n}(z)$ are positive. That the root of $B_{1}(z)$ is negative is then clear. That no two successive $B_{n}(z)$ have common roots follows from the well known identity

$$
A_{n}(z) B_{n-1}(z)-A_{n-1}(z) B_{n}(z)=(-1)^{n-1} z^{n}
$$

and the fact that $B_{n}(0)=1$ for all $n$. Now assume that the lemma holds for all $\nu$ up to and including $\nu=n-1$. Then

$$
\begin{aligned}
B_{n}\left(\rho_{\nu}^{(n-1)}\right) & =\left(1+d_{n} \rho_{\nu}^{(n-1)}\right) B_{n-1}\left(\rho_{\nu}^{(n-1)}\right)+\rho_{\nu}^{(n-1)} B_{n-2}\left(\rho_{\nu}^{(n-1)}\right) \\
& =\rho_{\nu}^{(n-1)} B_{n-2}\left(\rho_{\nu}^{(n-1)}\right) .
\end{aligned}
$$

Thus for two successive roots of $B_{n-1}$ the polynomial $B_{n}(z)$ takes on values with opposite signs. Hence there must be a root of $B_{n}$ between any two roots of $B_{n-1}$. Further, for the first root (in order of size) $\rho_{1}^{(n-1)}, B_{n}$ has a sign opposite to that of $B_{n-2}$ while for $z<0$ and sufficiently small the signs of the two polynomials are the same. Hence, since by the induction hypothesis there is no root of $B_{n-1}$ to the left of $\rho_{1}^{(n-1)}, B_{n}$ must have a root to the left of $\rho_{1}^{(n-1)}$. In a similar way $\left(B_{m}(0)=1\right.$, for all $\left.m\right)$ one shows that there must be a root of $B_{n}$ between $\rho_{n-1}^{(n-1)}$ and 0 . Thus all roots of $B_{n}$ are accounted for and the lemma is proved.

LEMMA 4.2. If $d_{\nu}>0$ for $\nu=0,1, \cdots, n$ then either $-1 / d_{n}$ is a root of $B_{n}$ or there is at least one root of $B_{n}$ to the left and at least one to the right of $-1 / d_{n}$. (Note that Lemma 4.1 insures that all roots of $B_{n}$ are real and negative.)

For $z=-1 / d_{n}$ one has

$$
B_{n}\left(-1 / d_{n}\right)=-1 / d_{n} B_{n-2}\left(-1 / d_{n}\right) .
$$

This expression may be equal to zero; if not one deduces from it that either $B_{n}$ or $B_{n-2}$ has a root to the left as well as to the right of $-1 / d_{n}$. An application of Lemma 4.1 completes the proof of this lemma.

A combination of these two lemmas leads to the following result.

LEMMA 4.3. If $d_{\nu}>0$ for $\nu=0,1, \cdots, n$ then there is at least one root of $B_{n}$ which is greater than or equal to every $-1 / d_{\nu}$ and at least one root which is less than or equal to every $-1 / d_{\nu}$.

If $\left\{d_{n}\right\}$ is unbounded there then exist polynomials $B_{n}(z)$ with arbitrarily large $n$ which have roots $\rho_{n}^{(n)}$ such that $\left|\rho_{n}^{(n)}\right|$ is less than any preassigned $\delta>0$. This completes the proof of Theorem 4.2. 
THEOREM 4.3. If $d_{n} \neq 0$ for all $n$ and if $\lim d_{n}=\infty$ then there does not exist a neighborhood of the origin in which the continued fraction (1.4) converges uniformly.

One easily verifies that

$$
g_{n}^{(n)}=\prod_{\nu=1}^{n} d_{\nu}=\prod_{\nu=1}^{n} 1 / \rho_{\nu}^{(n)} .
$$

If the conditions of the theorem are satisfied $\left(\left|\prod_{\nu=1}^{n} d_{\nu}\right|\right)^{1 / n}$ is unbounded. Now at least one of the quantities $\left|1 / \rho_{\nu}^{(n)}\right|$ is as large as the geometric mean of the set. The proof of the theorem is then completed by the same argument that was used in the two previous cases.

\section{BIBLIOGRAPHY}

1. Walter Leighton and W. T. Scott, A general continued fraction expansion, Bull. Amer. Math. Soc. vol. 45 (1939) pp. 596-605.

2. O. Perron, Die Lehre von den Kettenbrüchen, Leipzig, 1929.

3. W. J. Thron, Twin convergence regions for continued fractions $b_{0}+K\left(1 / b_{n}\right)$, Amer. J. Math. vol. 66 (1944) pp. 428-438.

WASHINGTON UNIVERSITY 